\title{
SYMPATHETIC LUMBAR PLEXUS BLOCK WITH BOTULINUM TOXIN IN THE TREATMENT OF ISCHEMIC PAIN
}

\author{
V.C. Paiva I , H.S. Fernandes I , H.A.Ashmawil , C.S. Roxol , K.L.L. Fonseca I , E.B. Espada I \\ I Department of Pain and Anaesthesiology from Hospital das Clínicas - University of Sao Paulo - HCFMUSP - São Paulo (Brazil)
}

Background: The thromboangiitis obliterans is an inflammatory peripheral arteriopathy, related to smoking. It involves of small and medium arteries of the upper and lower limbs with ischemic pain.

Case Report: A. B. J., 59 years old, male, with complaint of daily chronic ischemic pain in lower limbs by thromboangiitis obliterans. The patient was submitted on $06 / 18 / 16$ to a bilateral sympathetic lumbar plexus blockade with Ropivacaine, guided by radioscopy, with positive response. In 0I/03/20I7, a bilateral sympathetic lumbar plexus blockade was performed with injection of $10 \mathrm{ml}$ Ropivacaine $0.375 \%(37.5 \mathrm{mg})$ and I00IU of botulinum toxin (each side), guided by radioscopy, without intercurrences. The patient used, pre-procedure: transdermal patch of Fentanyl $25 \mathrm{mcg} / \mathrm{h}$, morphine LC $320 \mathrm{mg} /$ day (total oral Morphine equivalent dose: $380 \mathrm{mg} /$ day), Dipyrone $9000 \mathrm{mg} /$ day, Gabapentin $1200 \mathrm{mg} /$ day and Amitriptyline $50 \mathrm{mg} /$ day. The mean pre-procedure pain was $6.5 / 10$ per (numerical verbal scale), ranging from $4 / 10$ to $9 / 10$. The post-procedure pain intensity was $2 / 10$, with reported improvement of $60 \%$. After 30 days of the procedure, the patient showed lower pain intensity intensity: $5 / 10$.

Discussion: Lumbar sympathetic plexus block reduces vasoconstriction and improves peripheral circulation, thus improving pain in patients with peripheral vascular diseases. Due to its action (blocking the pre-synaptic acetylcholine release) botulinum toxin could act as a long acting sympathetic blocking.

References: I.Von Roenn, J. H., Paice, J.A., Preodor, M. E. CURRENT Diagnosis \& Treatment of Pain; 201 I, p.357.

2. Jabbari, B., Machado, D. Treatment of Refractory Pain with Botulinum Toxins - An Evidence-Based Review. Pain Medicine Journal, 20I I; 12: 1594-1606.
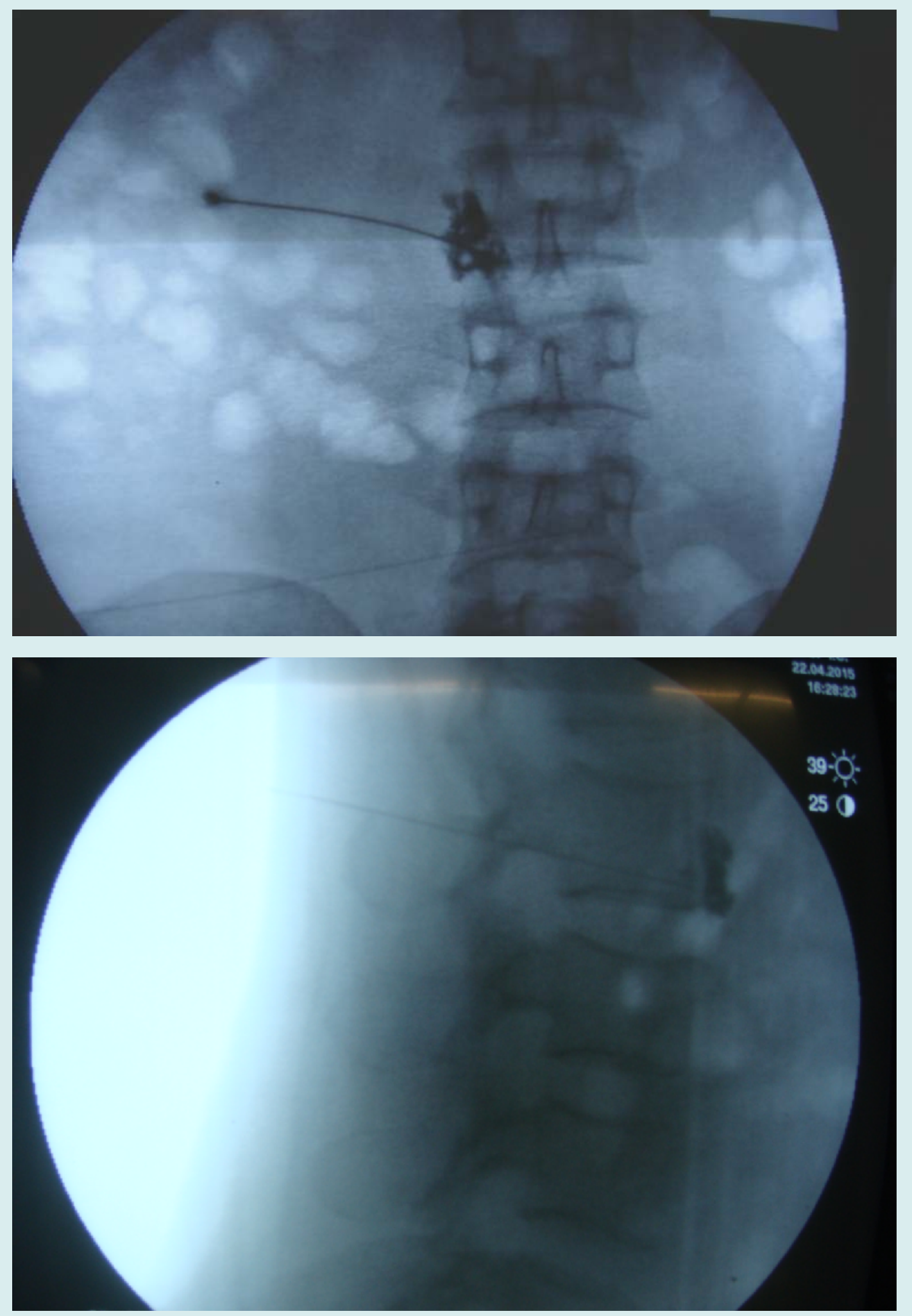

Learning points: The use of botulinum toxin to control pain in specific cases is already established and is now a possibility to be considered as a therapeutic alternative in difficult to control, requiring more studies that corroborate its efficacy and safety.

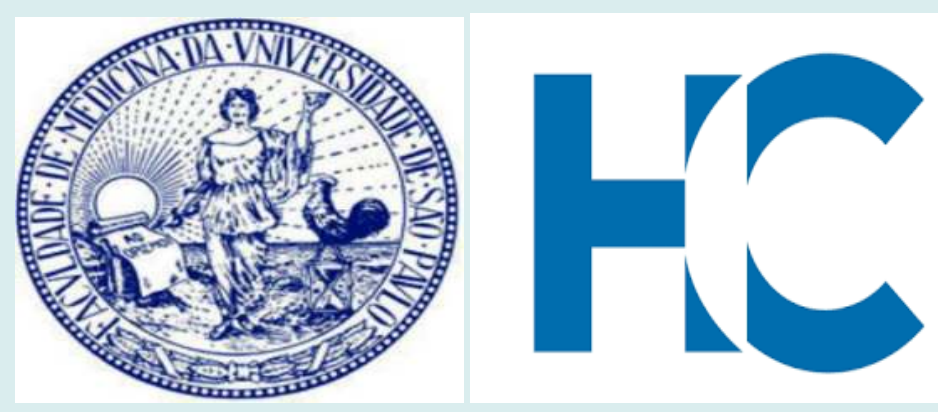

HOSPITAL DAS CLÍNICAS DA FACULDADE DE MEDICINAA DA UNIVERSIDADE DE SÃo PAULO 